\title{
The LXR-623-induced long non-coding RNA LINC01125 suppresses the proliferation of breast cancer cells via PTEN/AKT/p53 signaling pathway
}

\author{
Weijun Wan ${ }^{1}$, Yongying Hou', Ke Wang ${ }^{1}$, Yue Cheng ${ }^{1}$, Xia Pu ${ }^{1}$ and Xiufeng Ye ${ }^{1}$
}

\begin{abstract}
LXR-623 (WAY-252623), a liver X receptor agonist, reduces atherosclerotic plaque progression and remarkably inhibits the proliferation of glioblastoma cells, owing to its brain-penetrant ability. However, the role of LXR-623 against the proliferation of other cancer cells and the underlying mechanism remain unknown. Long non-coding RNAs (IncRNAs) serve as novel and crucial regulators that participate in cancer tumorigenesis and diverse biological processes. Here, we report a previously uncharacterized mechanism underlying IncRNA-mediated exocytosis of LXR-623 via the phosphatase and tensin homolog (PTEN)/protein kinase B (AKT)/p53 axis to suppress the proliferation of cancer cells in vitro. We found that LXR-623 significantly inhibited the proliferation and induced apoptosis and cell cycle arrest at $S$ phase in breast cancer cells in a concentration- and time-dependent manner. Experiments using a xenograft mouse model revealed the inhibitory effects of LXR-623 on tumor growth. We used IncRNA microarray to investigate the potential genes regulated by LXR-623. As a result, LINC01125 was found to be significantly upregulated in the cells treated with LXR-623. Gain- and loss-of-function assays were conducted to investigate the anti-proliferation role of LINC01125. LINC01125 knockdown resulted in the inhibition of the cytotoxic effect of LXR-623; in contrast, LINC01125 overexpression significantly enhanced the effect of LXR-623. LXR-623 and LINC01125-mediated anti-growth regulation is, at least in part, associated with the participation of the PTEN/AKT/mouse double minute 2 homolog (MDM2)/p53 pathway. In addition, SF1670, a specific PTEN inhibitor with prolonged intracellular retention, may strongly block the anti-proliferation effect induced by LXR-623 and LINC01125 overexpression. Chromatin immunoprecipitation (ChIP) assay results suggest that p53 binds to the promoter of LINC01125 to strengthen the expression of the PTEN/AKT pathway. Taken together, our findings suggest that LXR-623 possesses significant antitumor activity in breast cancer cells that is partly mediated through the upregulation in LINC01125 expression and enhancement in apoptosis via the PTEN/AKT/MDM2/p53 pathway.
\end{abstract}

\section{Introduction}

Breast cancer $(\mathrm{BC})$ is one of the most common cancers and accounts for about $30 \%$ of the cancer cases in females worldwide. It is ranked as the second most common cause of cancer-related deaths ${ }^{1,2}$. Treatment strategies for BC,

Correspondence: Xiufeng Ye (yxf1960@126.com)

'Department of Pathology, Chongqing Medical University, Chongqing 400016, China

Edited by E. Candi including breast-conserving surgery or mastectomy, chemotherapy, radiation therapy, hormone therapy, and other new therapies, are based on individual characteristics of clinical pathology ${ }^{3}$. However, many patients with $\mathrm{BC}$ experience relapse within a few years, and the longterm mortality rate remains high. Therefore, new therapeutic approaches and discovery of patient-friendly therapeutics that are safe and efficacious are desirable ${ }^{4,5}$.

\section{(c) The Author(s) 2019}

(c) (i) Open Access This article is licensed under a Creative Commons Attribution 4.0 International License, which permits use, sharing, adaptation, distribution and reproduction cc) in any medium or format, as long as you give appropriate credit to the original author(s) and the source, provide a link to the Creative Commons license, and indicate if changes were made. The images or other third party material in this article are included in the article's Creative Commons license, unless indicated otherwise in a credit line to the material. If material is not included in the article's Creative Commons license and your intended use is not permitted by statutory regulation or exceeds the permitted use, you will need to obtain permission directly from the copyright holder. To view a copy of this license, visit http://creativecommons.org/licenses/by/4.0/. 
Liver X receptors (LXRs) are nuclear receptors that induce the expression of the transporters responsible for promoting cholesterol efflux, leading to the reduction in atherosclerosis. LXRs are significant regulators of the fatty acid and glucose homeostasis as well as the immune system $^{6,7}$. Recent reports have revealed that blastic plasmacytoid dendritic neoplasm cell lines restored LXR target gene expression and increased cholesterol efflux via the upregulation in the expression of adenosine triphosphate-binding cassette $(\mathrm{ABC})$ transporters, $A B C A 1$ and $A B C G 1$, in response to $L X R$ agonist treatment ${ }^{8}$. In addition, LXR agonist may regulate the progression of prostate cancer through suppressor of cytokine signaling $3^{9}$ and reduce protein kinase B (Akt) phosphorylation in $\mathrm{BC}^{10}$. LXR-623, a novel LXR agonist and clinically effective anti-atherogenic agent, could significantly kill glioblastoma cells in an LXR $\beta$ - and cholesterol-dependent manner, cause tumor regression, and prolong the survival of mouse models, owing to its low toxicity and high brain-penetrant ability ${ }^{11}$. However, little is known about the antitumor impact of LXR-623 on other cancers.

Long non-coding RNAs (lncRNAs), a group of transcripts greater than 200 nucleotides in length, are involved in a variety of pathophysiological and biological processes in the human body, especially in the tumorigenesis and progression of cancer. Hence, IncRNAs have attracted the attention of researchers. Accumulating evidence indicates that the aberrant expression of lncRNAs is associated with tumorigenesis through multiple biological mechanisms involving epigenetic, transcriptional, and posttranscriptional alterations ${ }^{12-14}$. For instance, HOTAIR is a lncRNA that plays a key role in several cancers such as breast, gastric, colorectal, and cervical cancers and the expression level of HOTAIR is a potential biomarker for diagnostic and therapeutic purposes ${ }^{15,16}$.

Here, we hypothesize that lncRNAs may play a key role in the regulation of LXR-623-induced antitumor effects. In this study, we used $\mathrm{BC}$ cells and animal models to detect the antitumor activity of LXR-623 and investigated the underlying molecular mechanism. LXR-623 was shown to suppress the proliferation of $\mathrm{BC}$ cell lines and inhibit the growth of tumor xenografts. This action was associated with the expression of a lncRNA called LINC01125. Furthermore, the knockdown of LINC01125 blocked the inhibitory effects of LXR-623, whereas LINC01125 overexpression sensitized the BC cells to LXR-623. The results of the present study revealed that LINC01125 mediate the LXR-623-induced antiproliferation effect by regulating phosphatase and tensin homolog (PTEN) and AKT/p53 pathways. Therefore, this study provides a new insight into the chemopreventive mechanism associated with the LXR agonist application for cancer treatment.

\section{Results}

\section{LXR-623 suppresses the proliferation of BC cell lines}

To evaluate the effect of LXR-623 on BC cells and a normal epithelial breast cell line MCF-10A, we measured the cell viability using the cell counting kit 8 (CCK8) assay after the treatment of cells with LXR-623 at different concentrations $(1,2.5$, and $5 \mu \mathrm{M})$ for 24,48 , and $72 \mathrm{~h}$. As a result, we found that LXR-623 significantly suppressed the viability of MCF-7, MDA-MB-231, BT549, and MDAMB-453 cells in a dose- and time-dependent manner (Fig. 1a). Furthermore, flow cytometry was used to detect the number of apoptotic cells after exposure to LXR-623 at $5 \mu \mathrm{M}$ for 24,48 , and $72 \mathrm{~h}$. In comparison with the cells treated with dimethyl sulfoxide (DMSO; control group), MDA-MB-231 and BT549 cells treated with LXR-623 for $72 \mathrm{~h}$ showed almost $60 \%$ increase in the rate of apoptosis (Fig. 1b). Then, colony formation assay revealed the significant inhibition in the colony-forming ability of MDAMB-231 and BT549 cells after treatment with the tested drug at a concentration of $1,2.5$, and $5 \mu \mathrm{M}$ (Fig. 1c). These data suggest that LXR-623 could significantly inhibit proliferation and induce apoptosis in $\mathrm{BC}$ cells. We tested the expression of apoptosis-related proteins by western blot analysis and found that the expression of activated (cleaved) caspase-3 and BAX was upregulated and that of B-cell lymphoma 2 (BCL2) was downregulated in a timedependent manner (Fig. 1d). In addition, we investigated the effect of LXR-623 at $5 \mu \mathrm{M}$ concentration for 24,48 , and $72 \mathrm{~h}$ on cell cycle progression by flow cytometry and observed cell cycle arrest at the $\mathrm{S}$ phase in MDA-MB-231 and BT549 cells as compared to the control group (Figure S1a). The number of cells in the G1 stage decreased while that in the S stage increased in LXR-623-treated group versus the control group, indicating that the cells become insensitive to LXR-623-induced quiescence during $S$ phase. As LXR-623 may induce cell cycle arrest, we tested the protein levels of cyclin E1, cyclin-dependent kinase 2 (CDK2), and cyclin A2 with western blot analysis. As shown in Fig. 1c, the expressions of cyclin E1 and CDK2 were upregulated and cyclin A2 expression was downregulated, eventually manifesting the transition from the $\mathrm{G} 1$ to $S$ phase followed by quiescence in the $S$ phase in a time-dependent manner.

\section{Antitumor effects of LXR-623 in vivo}

To validate the antitumor effect of LXR-623 in vivo, female nude mice were subcutaneously injected with MDA-MB-231 cells to induce tumor formation. The mice were randomly divided into two groups once the tumor volumes reached about $100 \mathrm{~mm}^{3}$. During the treatment period of 10 days, tumor weight and volume significantly decreased for mice treated with LXR-623 as compared to those from the control group (Fig. 2a, b). LXR-623 could markedly inhibit the tumor growth (Fig. 2c). 


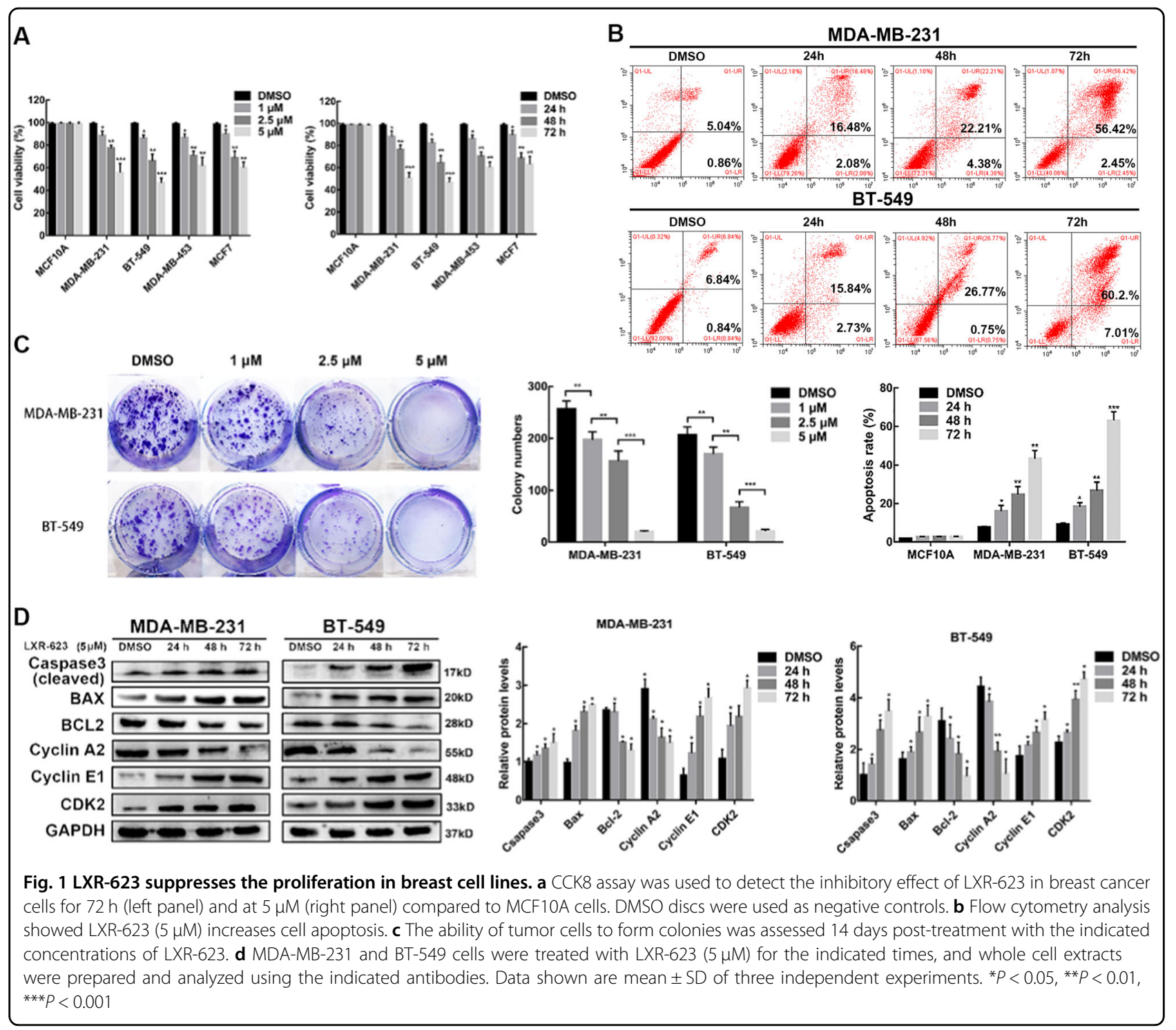

LXR-623-induced LINC01125 expression is downregulated in human $B C$ tissues and cells

To identify the candidate lncRNAs involved in LXR623-mediated tumor suppression, we used the IncRNA microarray to evaluate the differentially expressed genes regulated by LXR-623 at $5 \mu \mathrm{M}$ concentration for $48 \mathrm{~h}$ in MDA-MB-231 cells. IncRNA microarray results revealed significant changes in the expressions of 943 human lncRNAs after treatment with LXR-623. Of these human lncRNAs, 433 were upregulated and 510 were downregulated. $\mathrm{qRT}$-PCR results confirmed the increase in the expression of LINC01125 mediated by LXR-623 in the xenograft tumors as well as MDA-MB-231 and BT549 cells in a dose-dependent manner (Fig. 3a, b). LINC01125 expression level was downregulated in tumors as compared with the corresponding normal tissues (Fig. 3c). We used The Cancer Genome Atlas (TCGA) database to analyze the expression of LINC01125 (Fig. 3d) and found that LINC01125 expression was downregulated in BC tissues. qRT-PCR was used to confirm the downregulation of LINC01125 expression in MDA-MB-231, BT549, MCF-7, and MDA-MB-453 cells than in MCF$10 \mathrm{~A}$ cells. Immunofluorescence assay consistently showed that LINC01125 was more enriched in the cytoplasm of MDA-MB-231 and BT549 cells (Fig. 3e). Therefore, LINC01125 may play an important role as a suppressor gene in $\mathrm{BC}$.

\section{LINC01125 plays a critical role in LXR-623-induced apoptosis}

To assess the potential functional role of LINC01125 in LXR-623-induced tumor suppression, we examined the 


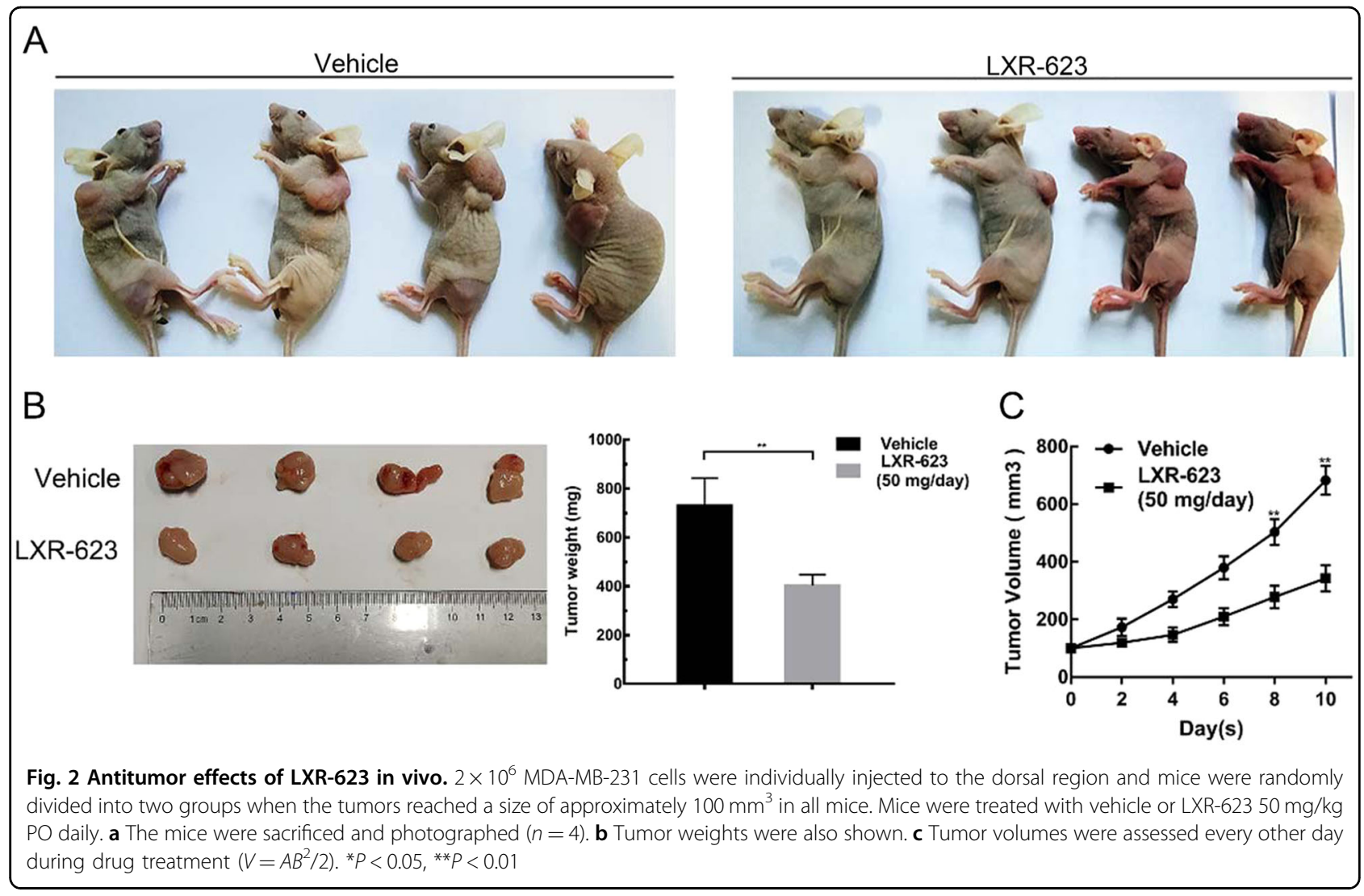

impact of LINC01125 overexpression and knockdown in $\mathrm{BC}$ cell lines. As shown in Fig. $4 \mathrm{a}, \mathrm{b}$, the knockdown of LINC01125 with a small interfering RNA (siRNA) led to an increase in the proliferation of tumors; however, the treatment of these transfected cells with LXR-623 $(5 \mu \mathrm{M})$ partially blocked the inhibitory effects of LXR-623, as revealed by the CCK8 and apoptosis assays. In contrast, cell viability significantly decreased for those cells overexpressing LINC01125 as compared with the negative control group. Upon treating cells with LXR-623, the apoptosis rate was dramatically increased. Western blotting results showed that LXR-623 induced the expression level of cleaved caspase- 3 and BAX and suppressed the expression of BCL2. This effect may be strengthened through the overexpression of LINC01125 or partly blocked through LINC01125 knockdown (Fig. 4c). Next, the knockdown of LINC01125 slightly reduced the number of cells in the S phase, but LINC01125 overexpression increased the number of cells in the $\mathrm{S}$ phase and strengthened the effect of LXR-623 (Figure S2a). Furthermore, western blot analysis showed that LXR-623induced upregulation in cyclin E1 and CDK2 expression and downregulation in cyclin A2 expression could be weakened through the knockdown of LINC01125 or magnified through the upregulation in LINC01125 expression (Fig. 4c).
LXR-623 inhibits cell proliferation through the activation of the LINC01125/PTEN/AKT/MDM2/p53 axis

To determine the mechanism underlying the antiproliferation effect of LXR-623 in vitro, we performed western blot analysis. The results showed that the cells treated with LXR-623 at $5 \mu \mathrm{M}$ concentration for 24,48 , and $72 \mathrm{~h}$ showed an increase in the expression of PTEN and p53 and reduced the expression of p-Akt and pMDM2 in a time-dependent manner (Fig. 5a). Furthermore, the knockdown of LINC01125 reduced the expression of PTEN and $\mathrm{p} 53$ and increased the expression of p-Akt and p-MDM2, while LINC01125 overexpression enhanced the expression of PTEN and p53 and lowered the expression of p-Akt and p-MDM2 (Fig. 5b). Immunohistochemistry (IHC) assay showed that the expressions of PTEN and p53 were increased in the xenograft tumors (Fig. 5c).

\section{SF1670 partially prevents the anti-proliferation effect of LXR-623 and LINC01125}

To examine the regulation of PTEN pathways mediated by LXR-623, MDA-MB-231 and BT549 cells were treated with a PTEN specific inhibitor (SF1670) for $48 \mathrm{~h}$. We tested the inhibitory effect of SF1670 at 1.5 and $3 \mu \mathrm{M}$ concentrations for $48 \mathrm{~h}$ by western blotting and found that SF1670 could block the expression of PTEN and p53 

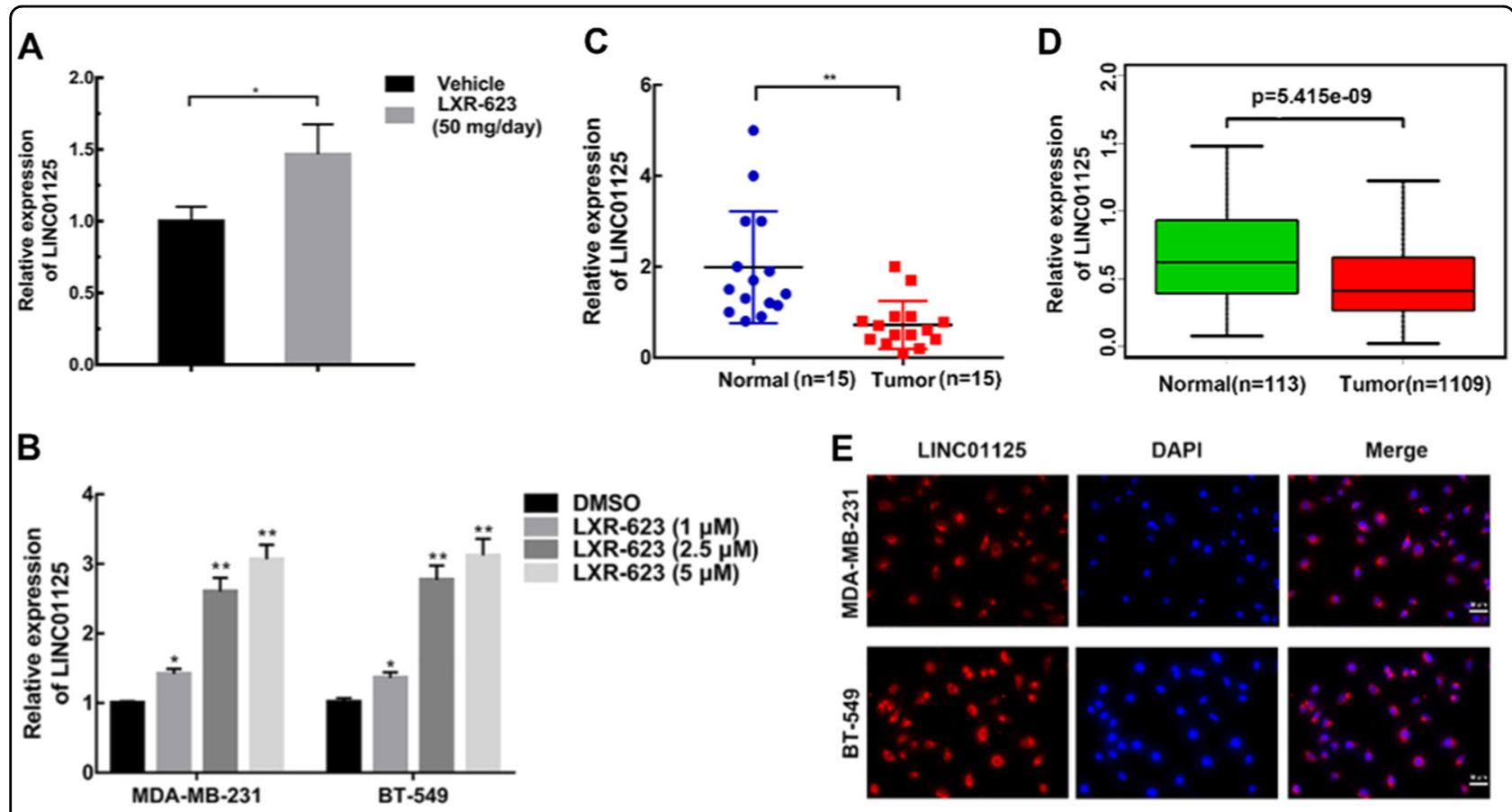

Fig. 3 LXR-623-induced LINC01125 is downregulated in human breast cancer tissues and cells. LncRNA array detected the differentially expressed genes regulated by LXR-623 at $5 \mu \mathrm{M}$ for $48 \mathrm{~h}$. a LINC01125 expression levels were measured in xenograft tumors and $\mathbf{b}$ in MDA-MB-231 and BT-549 cells treated with LXR-623 for $48 \mathrm{~h}$ at the indicated concentrations by RT-qPCR. c The expression of LINC01125 in the 15 pairs of breast cancer tissues and adjacent non-cancerous tissues by RT-qPCR. $\mathbf{d}$ TCGA database analysis of LINC01125 in breast cancer. e Representative images of FISH of LINC01125 in MDA-MB-231 and BT-549 (scale bar, $50 \mu \mathrm{m}$ ). ${ }^{*} P<0.05,{ }^{* *} P<0.01$

and increase the levels of p-AKT and p-MDM2 (Figure S3a). Then, SF1670 treatment at $3 \mu \mathrm{M}$ concentration could partly reverse the LXR-623-induced antiproliferation effect, as revealed from the results of the CCK8 assay and apoptosis experiments (Fig. 6a, b). We found an increase in the expression of cleaved caspase- 3 and BAX and a decrease in BCL-2 expression upon treatment with LXR-623. These effects of LXR-623 could be partly inhibited through SF1670 (Fig. 6c). The LINC01125-mediated anti-proliferation effect was also reversed by SF1670 (Fig. 6d, e). In addition, the BC cells treated with SF1670 showed an increase in the expressions of $\mathrm{p}-\mathrm{AKT}$ and $\mathrm{p}-\mathrm{MDM} 2$ and a decrease in the expressions of PTEN and p53 upon LINC01125 overexpression, suggestive of a link between LINC01125 and PTEN pathways (Fig. 6f).

\section{LINC01125 is a direct transcriptional target of p53}

To investigate the mechanism underlying the regulatory effect of LINC01125 on PTEN pathway, we examined the impact of p53 overexpression and knockdown on BC cell lines. Firstly, qRT-PCR results demonstrated that the expression of p53 was significantly induced or reduced in both MDA-MB-231 and BT549 cell lines after its overexpression or knockdown, respectively (Fig. 7a). CCK8 assay was used to evaluate the viability of the co-transfected MDA-MB-231 and BT549 cells. Treatment with LXR-623 had no effect on the co-transfection of LINC01125 overexpression vector and si-p53 as well as siLINC01125 and p53 overexpression vector (Fig. 7b). Furthermore, ChIP assay was performed to evaluate the binding of p53 to the promoter region of LINC01125, with an IgG-precipitated sample used as a negative control. We observed an increase in the binding between $\mathrm{p} 53$ and the LINC01125 promoter in the two cell lines (Fig. 7c). These data suggest that p53 showed significant binding to the promoters of LINC01125.

\section{Discussion}

Nuclear hormone receptor modulators could serve as potential cancer drugs. LXR modulators have gained attention owing to their actions against intestinal cancers, squamous cancer cells, BC cells, and prostate cancer cells $^{17-21}$. Previous studies have shown that an LXR agonist, T0901317, inhibited the proliferation of ovarian cancer cells and tumor formation through the elevated expression of p27, a CDK inhibitor ${ }^{22}$. The metabolic targets of LXRs and their ligands, including GW3965 compound, may also be related to the anti-proliferation effects observed in several cancer cells. This effect may be mediated through the elevated levels of the cholesterol transporter ATP-binding cassette (subfamily A [ABC1], 


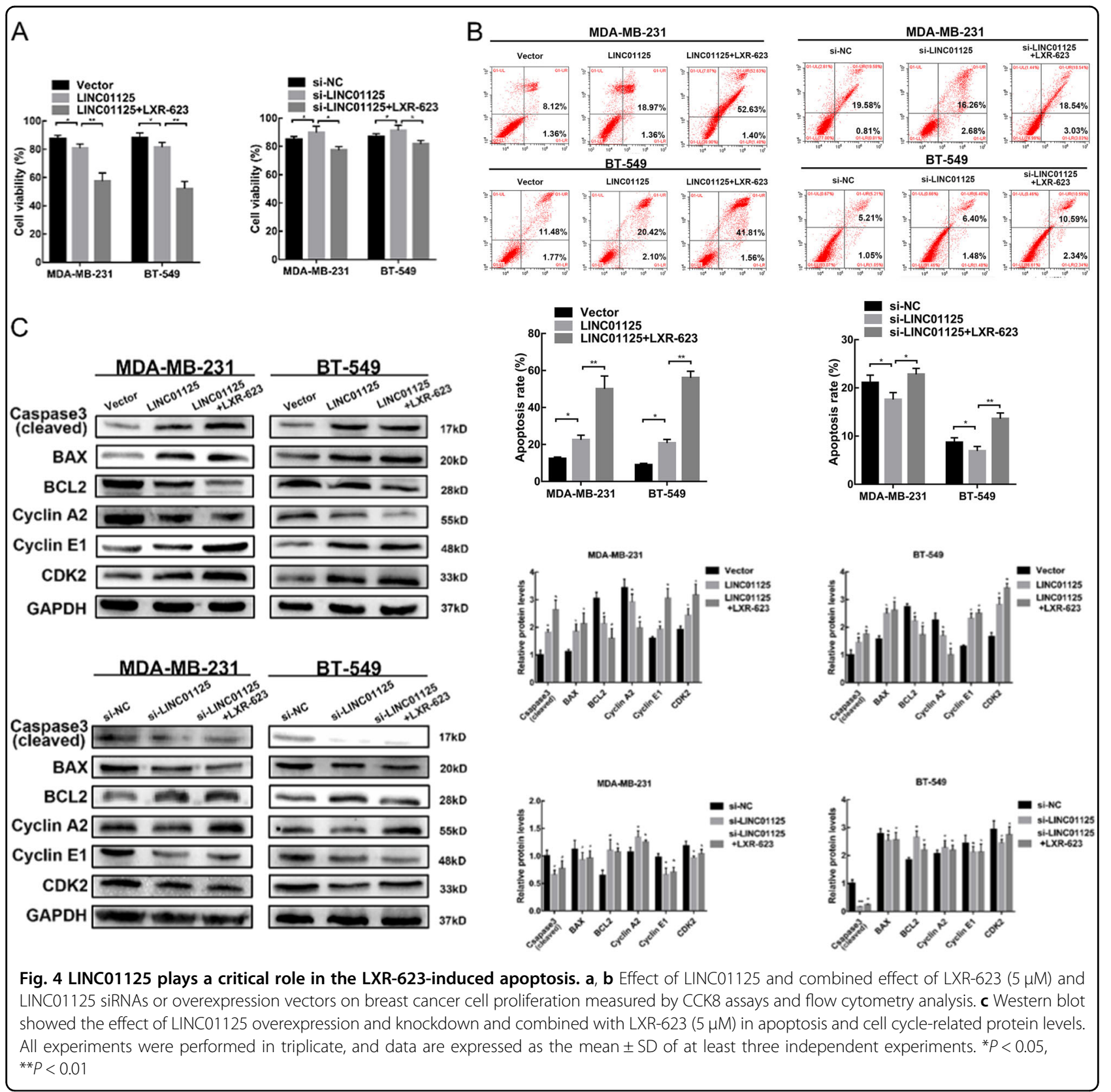

member 1 [ABCA1]) during carcinogenesis that inhibits low-density lipoprotein (LDL) uptake and induces cholesterol efflux ${ }^{11,23}$. Thus, targeting of LXRs with LXR agonists may serve as a strategy to suppress tumor growth.

LXR-623 is non-toxic and exerts anti-atherogenic activity on lipids. It was the first LXR agonist to enter clinical trials ${ }^{24}$. At present, little is known about the antitumor effect of LXR-623 and its underlying mechanism. To the best of our knowledge, this study is the first to show that LXR-623 may inhibit the proliferation of BC cells and regulate lncRNA expression. LXR-623 inhibits proliferation and induces apoptosis and cell cycle arrest in $\mathrm{BC}$ cells in a time- and concentration-dependent manner and suppresses tumor growth in xenograft mouse model, suggesting that LXR-623 may participate in the regulation of apoptosis-related pathways.

Evidences have indicated that lncRNAs are essential regulators involved in the control of the fundamental biological processes ${ }^{25-27}$. Here, we investigated lncRNA expression profiles in response to LXR-623 treatment in MDA-MB-231 cells and identified LXR-623-induced upregulated expression of the lncRNA, LINC01125. The analysis of TCGA database and tissues samples of patients 


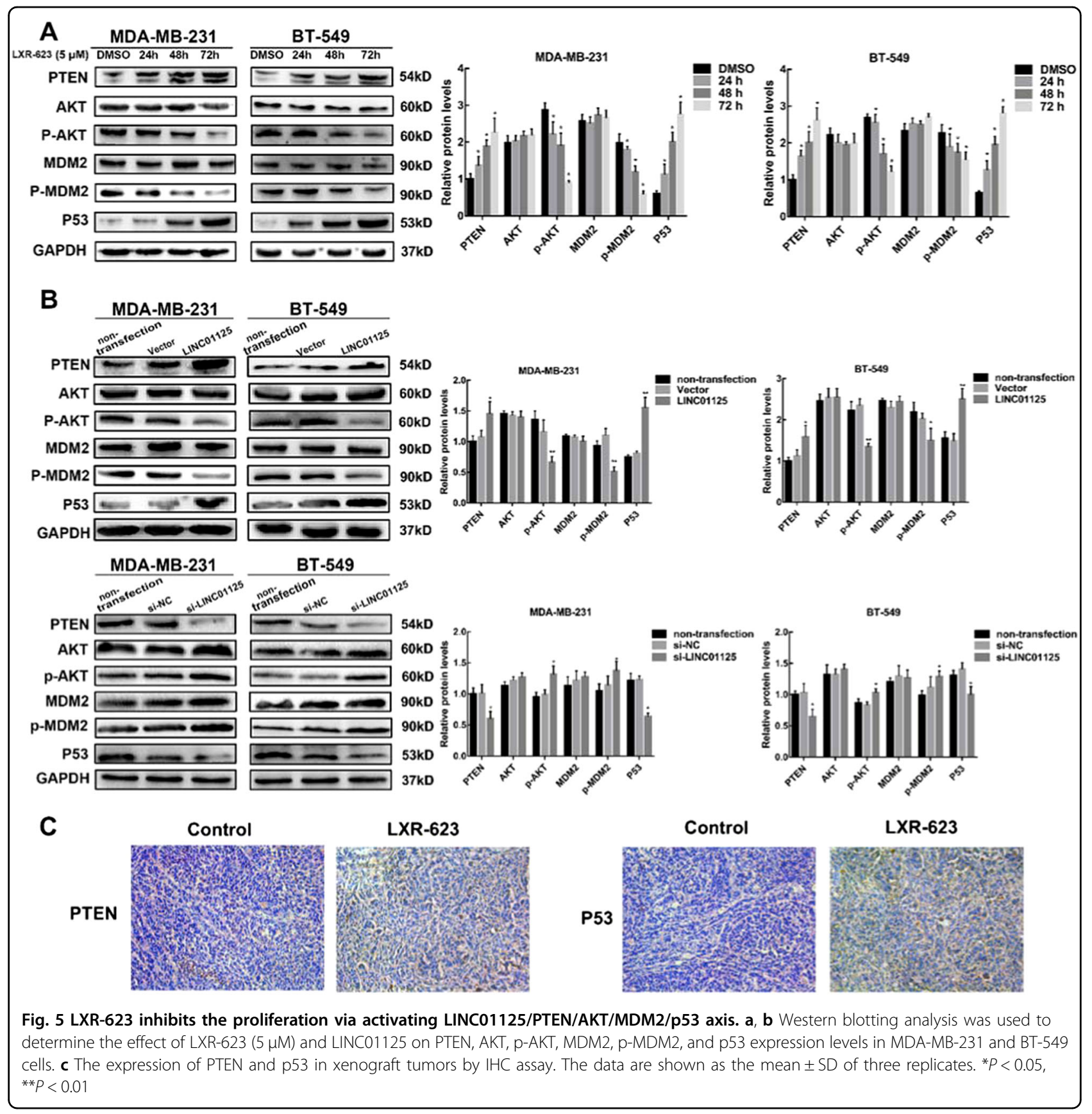

with $\mathrm{BC}$ demonstrated the significant downregulation in LINC01125 expression. The gain- and loss-of-function experiments showed that LINC01125 not only displays the characteristics of tumor suppressor gene but also blocks the inhibitory effects of LXR-623 on cell proliferation through the reduction in LINC01125 expression.

AKT signaling is a fundamental pathway that regulates multiple biological processes, including apoptosis and cell growth, and is known to be hyperactivated in various cancers $^{28}$. Kyoto Encyclopedia of Genes and Genomes
(KEGG) pathway analysis (Supplementary Figure S1B) demonstrated that the AKT pathway is associated with LXR-623-regulated differentially expressed lncRNAs. In addition, PTEN, a crucial tumor suppressor, is a prime antagonist of phosphatidylinositol-4,5-bisphosphate 3kinase (PI3K) and promotes AKT degradation. PTEN acts as a negative regulator of this pathway ${ }^{29-33}$. After treatment with SF1670, a PTEN inhibitor, the antiproliferative effect of LXR-623 was greatly suppressed. As LXR-623 and LINC01125 depend on the PTEN/AKT/ MDM2/p53 axis to suppress the proliferation of cancer 


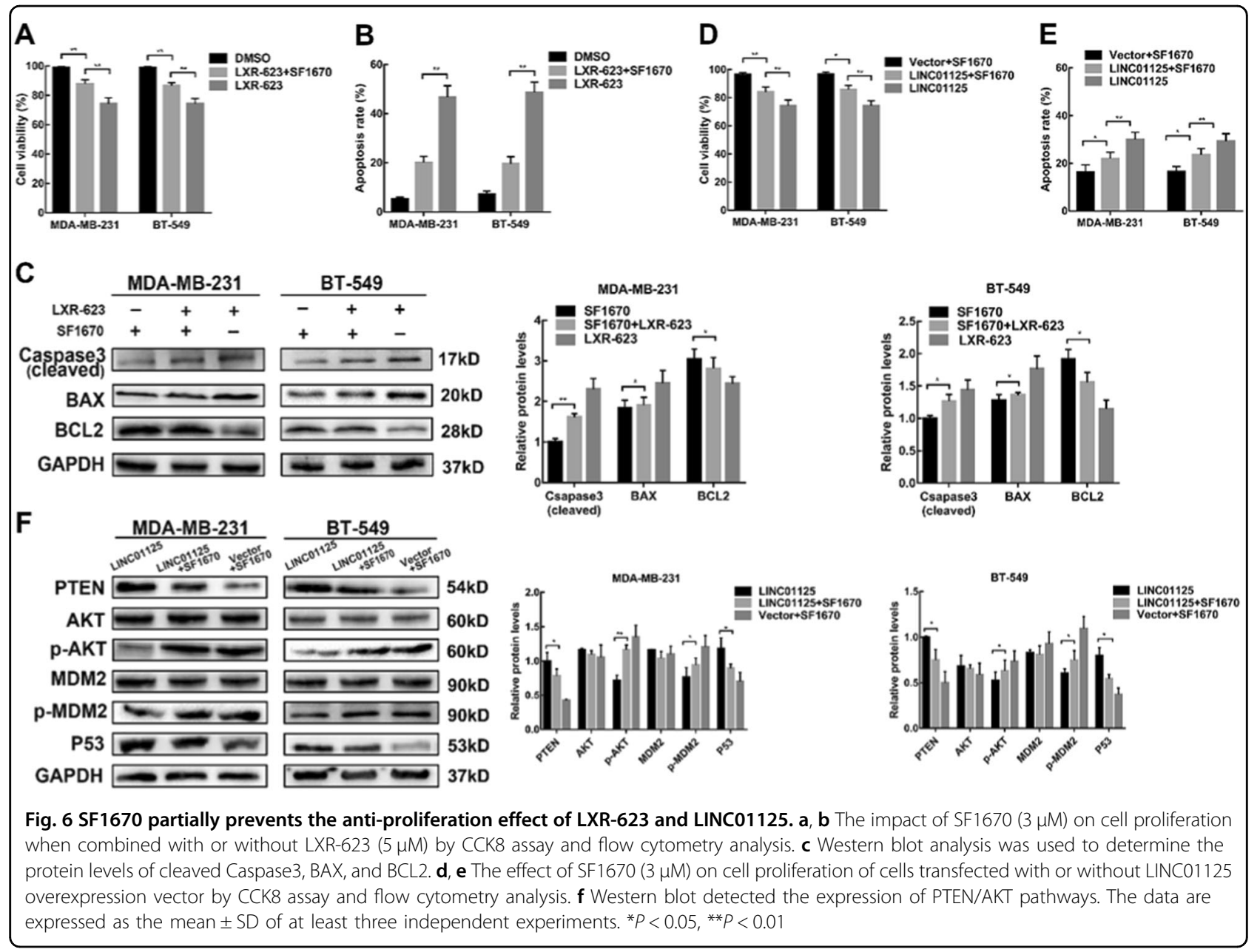

cells, it is important to understand the mechanism underlying the effect of LINC01125 on the PTEN/AKT/ MDM2/p53 pathway. We carried out a promoter prediction analysis of possible transcription factors that could regulate LINC01125 expression and found that the transcription factor, p53, was a good candidate. ChIP assay results also illustrated that LINC01125 was a direct transcriptional target of p53 in BC cell lines. Collectively, these results demonstrate that LINC01125 may serve as a coactivator of p53 to stimulate the PTEN pathway expression for mediating the LXR-623-induced antiproliferative effect.

Additional studies are warranted to evaluate the relationship between LXR-related lncRNAs and lipid metabolism in antitumor activities. The lncRNA LeXis was recently shown to promote cholesterol efflux and simultaneously block the cholesterol biosynthesis through the activation of LXRs ${ }^{34}$. Moreover, the metabolism of glucose, glutamine, and lipid is essential for tumor progression, and the tumor suppressor p53 is a transcription factor involved in cellular metabolism and appears to play a key role in tumor suppressive activities ${ }^{35}$. Whether
LXR-623-induced lncRNA regulates the expression of p53 transcription factors to modulate lipid metabolism may be investigated in future studies.

In our study, the LXR-623-p53-LINC01125-PTEN/ AKT axis showed promising antitumor effects on BC cells and provided a new insight into the chemopreventive mechanisms associated with LXR agonists. Further delineation of the molecular mechanisms underlying the effects of PTEN and AKT/p53 signaling on the differential modulation of tumor cell metabolism may improve the understanding of the link between genetic alterations and cellular metabolism in cancer and contribute to more effective and less toxic treatments.

\section{Materials and methods}

Cell culture, siRNA, plasmid construction, and transfection

The human cell lines MCF-10A, MDA-MB-231, and BT549 were obtained from the American Type Culture Collection (ATCC; Manassas, VA, USA), while MCF-7 and MDA-MB-453 cell lines were purchased from the Type Culture Collection of the Chinese Academy of Sciences (Shanghai, China). MDA-MB-231, MDA-MB-453, 


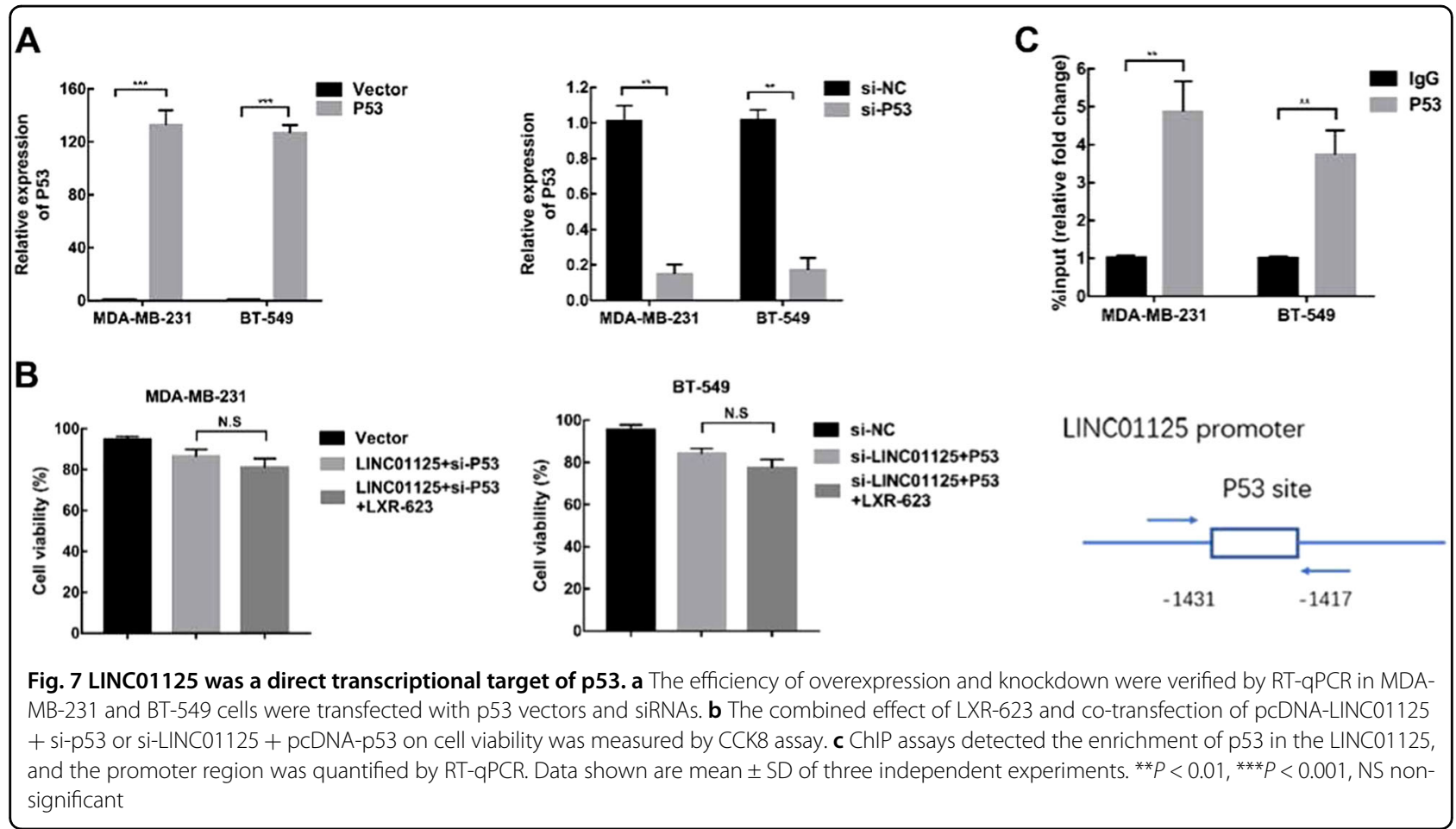

and MCF-7 cells were cultured in Dulbecco's modified Eagle's medium (DMEM; Gibco, Carlsbad, CA, USA) and BT549 cells were cultivated in Roswell Park Memorial Institute (RPMI)-1640 (Gibco) medium supplemented with $10 \%$ fetal bovine serum (FBS, Gibco). MCF-10A cells were cultured in MEBM BulletKit (Lonza, Basel, Switzerland). All cells were incubated in a humified $5 \% \mathrm{CO}_{2}$ incubator at $37^{\circ} \mathrm{C}$. Commercialized si-LINC01125, si-p53, and negative-control siRNA as well as pCDNA3.1LINC01125 and pCDNA3.1-p53 plasmid were purchased from Thermo Fisher Scientific (MA, USA). MDA-MB-231 and BT549 cells were seeded in six-well plates at a density of $3 \times 10^{5}$ cells/well overnight. Cell transfection was performed with Lipofectamine 2000 (Invitrogen, Carlsbad, CA, USA) according to the manufacturer's protocol. The sequences of siRNAs are presented in Supplementary Table S1. To knock down LINC01125, three siRNAs and a siRNA-NC were examined by qRT-PCR and siRNA-3 as the most effective one (Supplementary Figure S1A).

\section{Cell viability assay}

Cell viability was assessed with the CCK8 colorimetric assay (Bosterbio, Wuhan, China). Briefly, cells were initially transfected with an siRNA or indicated vector or co-transfected with siRNA and vector. At $48 \mathrm{~h}$ after transfection, cells $\left(2 \times 10^{3}\right.$ cells/well $)$ were cultured overnight in 96-well plates. The medium was replaced with that supplemented with LXR-623 or DMSO at indicated concentrations. After incubation for the indicated time,
$10 \mu \mathrm{L}$ of CCK8 reagent was added to each well and the cells were cultured for $2 \mathrm{~h}$ at $37^{\circ} \mathrm{C}$. The absorbance was recorded at $490 \mathrm{~nm}$ wavelength using a microplate reader (Multiskan ${ }^{\text {Tw }}$ GO microplate spectrophotometer; Thermo Fisher Scientific, Inc.). Cell viability was calculated according to the following formula: Cell viability $(\%)=$ (OD treatment - OD blank)/(OD control - OD blank).

\section{Apoptosis assay}

Annexin V-FITC/propidium iodide (PI) Apoptosis Detection Kit (Beyotime, Haimen, China) was used to study the mechanism of cell death according to the manufacturer's instructions after drug treatment or cell transfection. Cells $\left(5 \times 10^{5}\right.$ cells $\left./ \mathrm{mL}\right)$ were resuspended and mixed in $500 \mu \mathrm{L}$ of a binding buffer with $5 \mu \mathrm{L}$ of Annexin V-FITC and $5 \mu \mathrm{L}$ of PI. After incubation for $15 \mathrm{~min}$, cell apoptosis was tested by flow cytometry (NovoCyte, ACEA Biosciences, San Diego, CA, USA) and data analyses were performed by FlowJo.

\section{Cell cycle analysis}

To study the effect of LXR-623 and transfection with siRNA or indicated vector on cell cycle progression, cells were trypsinized and washed with phosphate-buffered saline (PBS), followed by treatment with $70 \%$ cold absolute ethanol (added drop-wise). The cells were incubated at $-20^{\circ} \mathrm{C}$ for at least for $24 \mathrm{~h}$ and then stained with $50 \mu \mathrm{g} /$ $\mathrm{mL}$ of PI (Sigma-Aldrich, St. Louis, USA) in the presence of $100 \mu \mathrm{g} / \mathrm{mL}$ RNAse (Sigma) at $37^{\circ} \mathrm{C}$ for $30 \mathrm{~min}$. The 
stained nuclei were analyzed on BD-FACSAria flow cytometer (BD Biosciences, CA, USA).

\section{Colony formation assay}

Cells were cultured in six-well plates $\left(0.5 \times 10^{3}\right.$ cells/ well) in a medium supplemented with LXR-623 at various concentrations $(1,2.5$, and $5 \mu \mathrm{M})$ or DMSO for 14 days, followed by treatment with $10 \%$ formaldehyde for $5 \mathrm{~min}$. Cells were stained with $1 \%$ crystal violet for $1 \mathrm{~min}$ before calculating the number of colonies.

\section{Xenograft tumor model}

All animal experiments were approved by the Chongqing Medical University Animal Care and Use Committee and performed in accordance with the guidelines of the National Institutes of Health. Female BALB/c mice (4-6-week-old, 18-20 g) were subcutaneously injected with MDA-MB-231 cells $\left(2 \times 10^{6}\right)$ in $100 \mu \mathrm{L}$ of sterile PBS in the dorsal region. Animals were randomly divided into two groups and treatment was started on the 2nd day. Once the tumors reached a measurable size of approximately $100 \mathrm{~mm}^{3}$ in all mice, mice were either treated with the vehicle $(0.5 \%$ methylcellulose, $2 \%$ Tween- 80 in water $)$ or LXR-623 $(50 \mathrm{mg} / \mathrm{kg})$ suspended in vehicle via oral gavage daily for 10 days. Tumor volume and body weight were evaluated every other day during the treatment period. Tumor volumes were measured with electronic digital calipers and determined by measuring length $(A)$ and width $(B)$ to calculate volume $\left(V=A B^{2} / 2\right)$.

\section{Microarray of IncRNA}

Total RNA was extracted from MDA-MB-231 cells treated with DMSO or LXR-623 $(5 \mu \mathrm{M})$ for $48 \mathrm{~h}$. Expression profiling of lncRNAs was performed using the Agilent human lncRNA array V.2.0 platform (Agilent Technologies, Santa Clara, CA, USA). The results of the microarray analysis were used for investigating the candidate target genes.

\section{Tissue sample}

The 15 pairs of $\mathrm{BC}$ tissues and adjacent non-cancerous tissues included in this study were obtained from the patients at the First Affiliated Hospital of Chongqing Medical University (Chongqing, China). None of the patients received chemotherapy or radiotherapy treatment before surgery. Tissues were collected after surgical resection and immediately frozen in liquid nitrogen and stored until further use. The present study was approved by the Ethics Committee of Chongqing Medical University and performed in accordance with the Declaration of Helsinki.

\section{RNA extraction, qRT-PCR, and western blot assays}

Total RNA was extracted from cells using TRIzol reagent (Invitrogen) and cDNAs were synthesized using the PrimeScript reverse transcriptase reagent kit (TaKaRa,
Shiga, Japan) following the manufacturer's instructions. Quantitative real-time PCR was carried out with SYBR Green $(\mathrm{TaKaRa})$ and the data were assessed on the CFX Connect Real-time System (BIO-RAD, CA, USA). Gene expression was evaluated with the $2^{-\triangle \Delta C T}$ method and the CT value was compared with that for glyceraldehyde3-phosphate (GAPDH). All experiments were repeated at least thrice. The primer sequences are shown in Supplementary Table 1.

Cells were washed twice with PBS and lysed in $100 \mu \mathrm{L}$ of radioimmunoprecipitation assay (RIPA) buffer (Bosterbio) containing protease inhibitors. Cell protein lysates were separated by $10 \%$ sodium dodecyl sulfate polyacrylamide gel electrophoresis (SDS-PAGE) and transferred onto $0.22-\mu \mathrm{m}$ polyvinylidene fluoride (PVDF) membranes (Sigma). The membranes were blocked with bovine serum albumin (BSA) for $2 \mathrm{~h}$ at room temperature and probed with rabbit primary antibodies (Supplementary Table 2). Then washed the membrane and followed by incubation with secondary antibodies (Cell Signaling Technology, Beverly, MA, USA) at room temperature for $1 \mathrm{~h}$. Densitometry (Quantity One software; Bio-Rad) was used to quantify the autoradiograms.

\section{Immunohistochemistry (IHC)}

For IHC, paraffin-embedded slides were deparaffinized, rehydrated, and stained with antibodies against PTEN (1:150) and p53 (1:150) at $4{ }^{\circ} \mathrm{C}$ overnight. The slides were treated with a secondary antibody, followed by incubation with 3,3'-diaminobenzidine (DAB). The slides were counter stained with hematoxylin and observed under a light microscope.

\section{RNA fluorescent in situ hybridization (FISH)}

RNA FISH assay was performed with a Fluorescent in Situ Hybridization Kit (RiboBio, China) following the manufacturer's suggestions. In order to achieve a sufficient signal-to-background ratio, multiple probes were targeted to each lncRNA sequence. A set of $15-20$ probes that covered the entire length of the RNA molecule provided the optimal signal strength, and each probe carried multiple fluorophores. The pooled FISH probes were resuspended at a final concentration of $25 \mu \mathrm{M}$ in an RNase-free storage buffer and protected from light at $-20^{\circ} \mathrm{C}$. The FISH results were captured using a confocal instrument (Leica TCS-SP8).

\section{Chromatin immunoprecipitation (ChIP)}

The ChIP assay was performed using the ChIP assay kit according to the manufacturer's protocol (Cell Signaling Technology). Cells were crosslinked with $1 \%$ (v/v) formaldehyde for $10 \mathrm{~min}$ at room temperature and the reaction was terminated with $125 \mathrm{mM}$ glycine treatment for $10 \mathrm{~min}$. The extracted chromatin was digested, 
sonicated, and fragmented into 150-900 bp. Chromatin extracts were immunoprecipitated with anti-p53 and antiIgG antibodies on Protein-A/G-Sepharose beads. After washing, elution, and de-crosslinking, PCR was performed using the primers spanning the putative p53-binding site on LINC01125 promoter.

\section{Chemicals}

LXR-623 and SF1670 were purchased from MedchemExpress (Monmouth Junction, NJ, USA).

\section{Statistical analysis}

All experimental assays were independently repeated in triplicates and data were expressed as mean \pm standard deviation (SD). Two-tailed Student's $t$-test and one-way analysis of variance (ANOVA) were used to assess the statistical difference between groups. All statistical data were evaluated using Statistical Program for Social Sciences 19.0 software (SPSS, Palo Alto, CA, USA) and presented with GraphPad Prism 7.0 (GraphPad Software, La Jolla, CA, USA). A value of $P$ less than 0.05 was considered as significant.

\section{Acknowledgements}

This work was supported by the Foundation for Science and Technology Research Project of Chongqing (CSTC2012ggB10002). The authors would like to thank the team of Dr. Chen from the Department of Cell Biology and Genetics, Chongqing Medical University for technical support. The important contributions of our colleagues in this research are gratefully acknowledged.

\section{Conflict of interest}

The authors declare that they have no conflict of interest.

\section{Publisher's note}

Springer Nature remains neutral with regard to jurisdictional claims in published maps and institutional affiliations.

Supplementary Information accompanies this paper at (https://doi.org/ 10.1038/s41419-019-1440-5).

Received: 27 September 2018 Revised: 9 January 2019 Accepted: 6 February 2019

Published online: 13 March 2019

\section{References}

1. Desantis, C. et al. Breast cancer statistics, 2013. CA Cancer J. Clin. 64, 52-62 (2014).

2. Lee, A. \& Djamgoz, M. B. A. Triple negative breast cancer: emerging therapeutic modalities and novel combination therapies. Cancer Treat. Rev. $\mathbf{6 2}$, 110-122 (2018).

3. Tong, C. W. S., Wu, M., Cho, W. C. S. \& To, K. K. W. Recent advances in the treatment of breast cancer. Front. Oncol. 8, 227 (2018).

4. Ranaware, A. M. et al. Magnolol: a Neolignan from the Magnolia family for the prevention and treatment of cancer. Int. J. Mol. Sci. 19, 2362 (2018).

5. Kyriakopoulou, K, Kefali, E., Piperigkou, Z., Bassiony, H. \& Karamanos, N. K. Advances in targeting epidermal growth factor receptor signaling pathway in mammary cancer. Cell. Signal. 51, 99-109 (2018).

6. Rasheed, A. \& Cummins, C. L. Beyond the foam cell: the role of LXRs in preventing atherogenesis. Int. J. Mol. Sci. 19, 2307 (2018).
7. Cermenati, G. et al. Liver $\mathrm{X}$ receptors, nervous system, and lipid metabolism. J. Endocrinol. Invest. 36, 435-443 (2013).

8. Ceroi, A. et al. LXR agonist treatment of blastic plasmacytoid dendritic cell neoplasm restores cholesterol efflux and triggers apoptosis. Blood $\mathbf{1 2 8}$ 2694-2707 (2016).

9. $\mathrm{Fu}, \mathrm{W}$. et al. LXR agonist regulates the carcinogenesis of PCa via the SOCS3 pathway. Cell. Physiol. Biochem. 33, 195-204 (2014).

10. Carbonnelle, D. et al. LXR activation down-regulates lipid raft markers FLOT2 and DHHC5 in MCF-7 breast cancer cells. Anticancer Res. 37, 4067-4073 (2017).

11. Villa, G. R. et al. An LXR-cholesterol axis creates a metabolic co-dependency for brain cancers. Cancer Cell. 30, 683-693 (2016).

12. Wei, M. M. \& Zhou, G. B. Long non-coding RNAs and their roles in non-smallcell lung cancer. Genomics Proteomics Bioinformatics 14, 280-288 (2016).

13. Virgilio, E. et al. Long non-coding RNAs in the gastric juice of gastric cancer patients. Pathol. Res. Pract. 214, 1239-1246 (2018).

14. Rashid, F., Shah, A. \& Shan, G. Long non-coding RNAs in the cytoplasm. Genomics Proteomics Bioinformatics 14, 73-80 (2016).

15. Hajjari, M. \& Salavaty, A. HOTAIR: an oncogenic long non-coding RNA in different cancers. Cancer Biol. Med. 12, 1-9 (2015).

16. Gupta, R. A. et al. Long non-coding RNA HOTAIR reprograms chromatin state to promote cancer metastasis. Nature 464, 1071-1076 (2010).

17. $\mathrm{Wu}, \mathrm{Y}$. et al. Liver $\mathrm{X}$ receptor as a drug target for the treatment of breast cancer. Anticancer Drugs 27, 373-382 (2016).

18. Chuu, C. P., Kokontis, J. M., Hiipakka, R. A. \& Liao, S. Modulation of liver X receptor signaling as novel therapy for prostate cancer. J. Biomed. Sci. 14, 543-553 (2007).

19. De Boussac, H. et al. Oxysterol receptors and their therapeutic applications in cancer conditions. Expert Opin. Ther. Targets 17, 1029-1038 (2013).

20. Lin, C. Y., Vedin, L. L. \& Steffensen, K. R. The emerging roles of liver $X$ receptors and their ligands in cancer. Expert Opin. Ther. Targets 20, 61-71 (2016).

21. Gronemeyer, H., Gustafsson, J. A. \& Laudet, V. Principles for modulation of the nuclear receptor superfamily. Nat. Rev. Drug Discov. 3, 950-964 (2004).

22. Rough, J. J., Monroy, M. A., Yerrum, S. \& Daly, J. M. Anti-proliferative effect of LXR agonist T0901317 in ovarian carcinoma cells. J. Ovarian Res. 3, 7686-7689 (2010).

23. Fukuchi, J., Kokontis, J. M., Hiipakka, R. A., Chuu, C. P. \& Liao, S. Antiproliferative effect of liver $X$ receptor agonists on LNCaP human prostate cancer cells. Cancer Res. 64, 7682-7685 (2004).

24. Katz, A. et al. Safety, pharmacokinetics, and pharmacodynamics of single doses of LXR-623, a novel liver X-receptor agonist, in healthy participants. J. Clin. Pharmacol. 49, 643-649 (2009).

25. Guo, $H$. et al. The aspirin-induced long non-coding RNA OLA1P2 blocks phosphorylated STAT3 homodimer formation. Genome Biol. 17, 24 (2016).

26. Zhu, J. et al. Long noncoding RNA MEG3 interacts with p53 protein and regulates partial p53 target genes in hepatoma cells. PLoS One 10, e0139790 (2015)

27. Wang, J., Wang, X., Chen, T., Jiang, L. \& Yang, Q. Huaier extract inhibits breast cancer progression through a LncRNA-H19/MiR-675-5p pathway. Cell. Physiol. Biochem. 44, 581-593 (2017).

28. Haddadi, N. et al. PTEN/PTENP1: 'Regulating the regulator of RTK-dependent PI3K/Akt signalling', new targets for cancer therapy. Mol. Cancer 17, 37 (2018).

29. Lim, H. J., Crowe, P. \& Yang, J. L. Current clinical regulation of PIJK/PTEN/Akt/ mTOR signalling in treatment of human cancer. J. Cancer Res. Clin. Oncol. 141, 671-689 (2015).

30. Martelli, A. M. et al. Targeting the translational apparatus to improve leukemia therapy: roles of the PI3K/PTEN/Akt/mTOR pathway. Leukemia $\mathbf{2 5}$, 1064-1079 (2011).

31. Jamaspishvili, T. et al. Clinical implications of PTEN loss in prostate cancer. Nat. Rev. Urol. 15, 222-234 (2018).

32. Lu, Y. M., Cheng, F. \& Teng, L. S. The association between phosphatase and tensin homolog hypermethylation and patients with breast cancer, a metaanalysis and literature review. Sci. Rep. 6, 32723 (2016).

33. Pan, S. et al. Decreased expression of ARHGAP15 promotes the development of colorectal cancer through PTEN/AKT/FOXO1 axis. Cell Death Dis. $\mathbf{9}$, 673 (2018).

34. Sallam, T. et al. Feedback modulation of cholesterol metabolism by the lipidresponsive non-coding RNA LeXis. Nature 534, 7605 (2016).

35. Parrales, A. \& Iwakuma, T. p53 as a regulator of lipid metabolism in cancer. Int J. Mol. Sci. 17, 2074 (2016) 